\title{
Galaxies in the first billion years: implications for re-ionization and the star formation history at $z>6$
}

\author{
Andrew J. Bunker ${ }^{1}$, Elizabeth R. Stanway ${ }^{2}$, Laurence P. Eyles ${ }^{1}$, \\ Richard S. Ellis ${ }^{3}$, Richard G. McMahon ${ }^{4}$, Mark Lacy ${ }^{5}$ and \\ Daniel P. Stark ${ }^{3}$ \\ ${ }^{1}$ University of Exeter, School of Physics, Stocker Road, Exeter, EX4 4QL, UK \\ email: bunker@astro.ex.ac.uk \\ ${ }^{2}$ Astronomy Dept., University of Wisconsin, 475 N. Charter Street, Madison, WI 53706, USA \\ ${ }^{3}$ California Institute of Technology, Mail Stop 169-327, Pasadena, CA 91109, USA \\ ${ }^{4}$ Institute of Astronomy, Madingley Road, Cambridge, CB3 0HA, UK \\ ${ }^{5}$ Spitzer Science Center, 1200 E. California Blvd., Pasadena, CA 91125, USA
}

\begin{abstract}
We discuss the selection of star-forming galaxies at $z \simeq 6$ through the Lyman-break technique. Spitzer imaging implies many of these contain older stellar populations ( $>200 \mathrm{Myr}$ ) which produce detectable Balmer breaks. The ages and stellar masses $\left(\sim 10^{10} \mathrm{M}_{\odot}\right)$ imply that the star formation rate density at earlier epochs may have been significantly higher than at $z \simeq 6$, and might have played a key role in re-ionizing the universe.
\end{abstract}

Keywords. galaxies: high-redshift; evolution; formation; stellar content

We have discovered a population of star-forming galaxies at $z \simeq 6$ and beyond (within the first billion years) through the $i^{\prime}$-drop technique. The first application of this to HSTACS imaging was presented in Stanway et al. (2003), using the public GOODS survey. We were able to prove this technique through Keck-DEIMOs spectroscopy (Bunker et al. 2003). Using the same $i^{\prime}$-drop selection, our first analysis of the Hubble Deep Field revealed 50 star forming galaxies at redshifts around six with magnitudes $z_{A B}>28.5$ (Bunker et al. 2004). Spitzer observations with IRAC enable us to estimate the stellar masses and luminosity-weighted ages for this population; we find in some cases that there are Balmer breaks, indicating ages of $>200 \mathrm{Myr}$ and formation redshifts of $z \simeq 10$ (Eyles et al. 2005). From the whole sample of $v$-drops and $i^{\prime}$-drops we estimate the stellar mass density at $z \approx 5$ (Stark et al. 2007) and at $z \approx 6$ (Eyles et al. 2007). The implications of this work are that the previous star formation history was higher prior to $z \simeq 6$, and might have played a key role in generating the UV photons necessary to re-ionize the universe at $z \simeq 8-10$. Our work is the strongest constraint to date on the star formation history at $z>6$.

\section{References}

Bunker, A. J., Stanway, E. R., Ellis, R. S., et al. 2003, MNRAS (Letters), 342, L47

Bunker, A. J., Stanway, E. R., Ellis, R. S., \& McMahon, R. G. 2004, MNRAS, 355, 374

Eyles, L. P, Bunker, A. J., Stanway, E. R., et al. 2005, MNRAS, 364, 443

Eyles, L. P, Bunker, A. J., Ellis, R. S., et al. 2006, MNRAS, 374, 910

Stanway, E. R., Bunker, A. J., \& McMahon, R. G. 2003, MNRAS, 342, 439

Stark, D. P., Bunker, A. J., Ellis, R. S., Eyles, L. P., \& Lacy, M. 2007, ApJ, 659, 84 